Pacific Journal of Mathematics

ON UNIQUENESS QUESTIONS FOR HYPERBOLIC 


\title{
ON UNIQUENESS QUESTIONS FOR HYPERBOLIC DIFFERENTIAL EQUATIONS
}

\author{
JoHN P. ShanahaN
}

1. Statement of results. This note is concerned with the existence, uniqueness, and successive approximations for solutions of the initial value problem

$$
z_{x y}=f(x, y, z, p, q), z(x, 0)=\sigma(x), z(0, y)=\tau(y),
$$

where $\sigma(0)=\tau(0)=z_{0}$, on a rectangle $R: 0 \leqq x \leqq a, 0 \leqq y \leqq b$. By a solution is meant a continuous function having partial derivatives almost everywhere and satisfying the integral equation

$$
z(x, y)=\sigma(x)+\tau(y)-z_{0}+\int_{0}^{x} \int_{0}^{y} f\left(s, t, z(s, t), z_{x}(s, t), z_{y}(s, t)\right) d s d t .
$$

Actually it will be clear from the conditions imposed on $\sigma, \tau$ and $f$ that any solution of (1) is uniformly Lipschitz continuous. Let $D$ be the five-dimensional set $D=\{(x, y, z, p, q):,(x, y) \in R$ and $z, p, q$ arbitrary $\}$. Let $f(x, y, z, p, q)$ be defined and continuous on $D$, such that $\mid f(x, y, z$, $p, q,) \mid<N=$ const. for $(x, y, z, p, q) \in D$. Let $\sigma(x), \tau(y)$ be defined and uniformly Lipschitz continuous on $0 \leqq x \leqq a, 0 \leqq y \leqq b$, respectively (so that $|\sigma(x)-\sigma(\bar{x})| \leqq K|x-\bar{x}|,|\tau(\mathrm{y})-\tau(\bar{y})| \leqq K|y-\bar{y}|$ for some constant $K$ ) and let $\sigma(0)=\tau(0)=z_{0}$. In addition, for $(x, y) \in R$ and arbitrary $z, p, q, \bar{z}, \bar{p}, \bar{q}$ assume that

$$
|f(x, y, z, p, q,)-f(x, y, \bar{z}, \bar{p}, \bar{q})| \leqq \varphi(x, y,|z-\bar{z}|,|p-\bar{p}|,|q-\bar{q}|),
$$

where $\varphi(x, y, z, p, q)$ is a continuous, non-negative function defined for $(x, y) \in R$ and non-negative $z, p, q$, non-decreasing in each of the variables $z, p, q$, and with the property that for every $(\alpha, \beta)$, where $0<\alpha \leqq a, 0<\beta \leqq b$, the only solution of

$$
z(x, y)=\int_{0}^{x} \int_{0}^{y} \varphi\left(s, t, z(s, t), z_{x}(s, t), z_{y}(s, t)\right) d s d t
$$

in the rectangle $R_{\alpha \beta}: 0 \leqq x \leqq \alpha, 0 \leqq y \leqq \beta$ is $z \equiv 0$.

TheOREM (*). Under the above assumptions on $\sigma, \tau, f$ and $\phi,(1)$ possesses one and only one solution on $R$. This solution is the uniform limit of the successive approximations defined by

Received June 25, 1959. This research was supported by the United States Air Force through the Air Force Office of Scientific Research of the Air Research and Development Command, under contract No. AF 18 (603)-41. Reproduction in whole or in part is permitted for any purpose of the United States Government. 


$$
z_{0}(x, y)=\sigma(x)+\tau(y)-z_{0}
$$

and, for $n=1,2,3, \cdots$, by

$\left(4_{n}\right) \quad z_{n}(x, y)=z_{0}(x, y)+\int_{0}^{x} \int_{0}^{y} f\left(x, y, z_{n-1}(s, t), z_{n-1} x(s, t), z_{n-1}(s, t)\right) d s d t$.

The existence assertion of $(*)$ neither implies nor is implied by that in Hartman-Wintner [3] and its generalizations due to Conti, Szmydt, Ciliberto, Kisynski (for references, see [6] and [2]). The uniqueness assertion of $(*)$ can be considered as a crude analogue of Kamke's uniqueness theorem (cf. [5], p. 139) in the theory of ordinary differential equations. Finally, the assertion concerning the convergence of successive approximations is an analogue of a result on ordinary differential equations (cf. Viswanatham [8] and references there to van Kampen, to Wintner and to Dieudonne, and Coddington and Levinson [1]).

A theorem similar to (*), in which $f$ and $\varphi$ do not depend on $p, q$ is proved by Guglielmino [2]. The proof of $(*)$ below will be a generalization of that of [2]. A uniqueness theorem for (1) involving a majorant function of the form $\varphi(z, p, q)=\varphi(|z|+|p|+|q|)$ is given in [6]. (After the completion of this manuscript, I learned" of a paper "On the existence theorem of Caratheodory for ordinary and hyperbolic differential equations" by W. Walter, written at about the same time, which contains a theorem in the direction of the uniqueness assertion of (*). Walter's assumptions, however, are somewhat different.)

REMARK. It will be clear from the proofs that $(*)$ remains valid if $f, z, p, q, \sigma, \tau$ are n-vectors (say, with the norm $|z|=\sum_{k=1}^{n}\left|z^{k}\right|$ or $|z|=\max \left(\left|z^{1}\right|, \cdots,\left|z^{n}\right|\right)$ if $\left.z=\left(z^{1}, \cdots, z^{n}\right)\right)$. Of course $\rho$ will still be a function of 5 variables, (not of $(3 n+2)$ variables as $f$ is).

A theorem suggested by Nagumo's uniqueness theorem (cf. [5], p. 97) for ordinary differential equations is the following:

Theorem (**). Let $f(x, y, z, p, q)$ be defined, continuous and bounded on $D$, and satisfy, for $x y>0$ and arbitrary $z, p, q, \bar{z}, \bar{p}, \bar{q}$,

$$
\begin{aligned}
\mid f(x, y, z, p, q,)-f(x, y, \bar{z}, \bar{p}, \bar{q}) \leqq & c_{1}(x, y)|z-\bar{z}| \mid x y+ \\
& c(x, y)|p-\bar{p}|\left|y+c_{3}(x, y)\right| q-\bar{q}|| x,
\end{aligned}
$$

where $c_{i}(x, y), i=1,2,3$, are non-negative, continuous functions such that

$$
c_{1}+c_{2}+c_{3} \equiv 1 \text {. }
$$

Let $\sigma(x), \tau(y)$ be as in (*), and, in addition, let

1 Added in proof, 4 April 1960. Since this paper was accepted for publication, the following related articles have appeared: W. L. Walter, Ueber die Differentialgleichung $u_{x y}=f\left(x, y, u, u_{x}, u_{y}\right)$, I and II, Math. Zeit., 71 (1959), 308-324 and 436-453; my attention has also been called to the paper of J. B. Diaz and W. L. Walter, On uniqueness theorems for ordinary differential equations and for partial differentiale equatitions of hyperbolic type, to appear in Trans. A.M.S.. 


$$
\sigma_{x}(+0)=\lim _{x \rightarrow+0} \sigma_{x}(x), \tau_{y}(+0)=\lim _{y \rightarrow+0} \tau_{y}(y)
$$

exist. Then (1) has at most one solution $z=z(x, y)$. Furthermore, if

$$
c_{1}(0,0)>0,
$$

then a solution exist and is the uniform limit of the successive approximations (4).

In (6), $x\left[\right.$ or $y$ ] tends to +0 through the set of values on which $\sigma_{x}$ [or $\tau_{y}$ ] exists.

Nagumo's theorem follows from Kamke's (with $\varphi(x, y)=y / x)$. However (**) does not follow from $(*)$ because $\varphi(x, y, z, p, q)$ is assumed continuous on $x=0$ and on $y=0$.

REMARK 1. (**) is valid if $f, z, p, q, \sigma, \tau$ are $n$-vectors (say $z=$ $\left(z^{1}, \cdots, z^{n}\right)$ and either $|z|=\sum_{k=1}^{n}\left|z^{k}\right|$ or $\left.|z|=\max \left(\left|z^{1}\right|, \cdots,\left|z^{n}\right|\right)\right)$.

REMARK 2. A modification of an example of Perron [7] in the theory of ordinary differential equations will show that $(* *)$ is false if $c_{1}=$ const. $>1, c_{2} \equiv c_{3} \equiv 0$ (so that $f$ does not depend on $p, q$ ). Also, a modification of an example of Haviland [4] shows that successive approximations need not converge if $c_{1}=$ const. $>1, c_{2}=c_{3} \equiv 0$.

The proof of $(*)$ will be given in $\S \S 2-4$ below; that of $(* *)$ in $\S \S 5-6$; finally, the proof of the last remark will be indicated in $\$ 7$.

The results above answer some questions suggested by Professor P. Hartman. I also wish the acknowledge helpful discussions with him.

2. Proof of $(*)$. Preliminaries. In the proof of $(*)$ below, there is no loss of generality in supposing that $\varphi$ is bounded, say $0 \leqq \varphi(x, y$ $z, p, q,) \leqq 2 N$ on $D$. For otherwise $\phi$ can be replaced by $\bar{\varphi}$, where $\overline{\mathcal{P}}(x, y, z, p, q)$ equals $\mathcal{q}(x, y, z, p, q)$ or $2 N$ according as $\varphi(x, y, z, p, q)$ does not or does exceed $2 N$. It is clear that $\bar{\rho}$ is continuous and nondecreasing in each of the variables $z, p, q$. Furthermore, the only solution $z(x, y)$ of

$$
z(x, y)=\int_{0}^{x} \int_{0}^{y} \bar{\varphi}\left(s, t, x(s, t), z_{x}(s, t,), z_{y}(s, t)\right) d s d t
$$

on any rectangle $R_{\alpha \beta}: 0 \leqq x \leqq \alpha(\leqq a), 0 \leqq y \leqq \beta(\leqq b)$ is $z \equiv 0$.

In order to see this, note that $\varphi(x, y, 0,0,0) \equiv 0$ because $z=0$ is a solution of (3). Hence there exists an $\varepsilon>0$ such that $0 \leqq \varphi(x, y, z$, $p, q) \leqq 2 N$ if $|z|,|p|,|q|<\varepsilon$. Suppose that $z(x, y) \neq \equiv$ is a solution of (3') on $R_{\alpha \beta}$. Let $d, 0 \leqq d \leqq\left(\alpha^{2}+\beta^{2}\right)^{\frac{1}{2}}$, be the largest value of $r$ for which $z(x, y) \equiv 0$ in the intersection $S_{r}$ of $x^{2}+y^{2} \leqq r^{2}$ and $R_{\alpha \beta}$. If $U$ is any neighborhood of $S_{d}$ (relative to $R_{\alpha \beta}$ ), there exists a rectangle $R_{\gamma \delta}$ in $U$ on which $z \not \equiv 0$. Since $z \equiv 0$ on $S_{d}$, it is clear that if $U$ is "sufficiently small", then, on $U$ (hence on $R_{\gamma \delta}$ ), $|z|<\varepsilon$ and, almost everywhere, $\left|z_{x}\right|+\left|z_{y}\right|<\varepsilon$. But then $z \not \equiv 0$ is a solution of (3) on $R_{\gamma \delta}$. Since this is impossible, the only solution of $\left(3^{\prime}\right)$ on $R_{\alpha \beta}$ is $z \equiv 0$. 
It will be convenient to have the following notation. $R_{1}$ denotes a subset (not always the same) of $R$ of the from $E \times[0, b]$, where $E$ is a (Lebesgue) measurable subset of $[0, a]$ with means $E=a$. Similary, $R_{2}$ is a subset (not always the same) of the form $[0, a] \times E$, where $E$ is a measurable subset of $[0, b]$ and means $E=b$. Partial derivatives $z_{x}, z_{y}$ of a function $z$ will be denoted by $p, q$.

3. Lemma for $(*)$. The proof of $(*)$ will depend on the following lemma.

Lemma 1. Let $\alpha(x, y), \beta(x, y) \gamma(x, y)$ be non-negative, measurable functions defined on $R, R_{1}, R_{2}$, respectively, such that $\alpha$ is continuous, $\beta$ is uniformly Lipschitz continuous with respect to $y$ and $\gamma$ is uniformly Lipschitz continuous with respect to $x$, In addition, let

$$
\begin{aligned}
& \alpha(x, y) \leqq \int_{0}^{x} \int_{0}^{y} \varphi(s, t, \alpha(s, t), \beta(s, t), \gamma(s, t)) d s d t, \\
& \beta(x, y) \leqq \int_{0}^{y} \varphi(s, t, \alpha(x, t), \beta(x, t), \gamma(x, t)) d t, \\
& \gamma(x, y) \leqq \int_{0}^{x} \varphi(s, y, \alpha(s, y), \beta(s, y), \gamma(s, y)) d s,
\end{aligned}
$$

where $\phi$ satisfies the conditions of $(*)$ and is bounded. Then $\alpha \equiv \beta \equiv$ $\gamma \equiv 0$.

Note that the Lipschitz continuity of $\beta$ [or $\alpha$ ] with respect to $y$ [or $x$ ] is assumed to be uniform with respect to $x$ and $y$.

The proof of the lemma below follows a suggestion made by $R$. Sacksteder. My original proof, which will be omitted, depended on two results. The first result is an existence theorem for

$$
z(x, y)=\psi(x, y)+\int_{0}^{x} \int_{0}^{y} \varphi(s, t, z(s, t), p(s, t), q(s, t)) d s d t,
$$

where $\psi$ is a non-negative, uniformly Lipschitz continuous function which is non-decreasing in $x$ and in $y$. This existence theorem is proved by using the successive approximations $z_{0}=\psi(x, y)$ and

$$
z_{n}(x, y)=z_{0}(x, y)+\int_{0}^{x} \int_{0}^{y} \varphi\left(s, t, z_{n-1}, p_{n-1}, q_{n-1}\right) d s d t
$$

which satisfy

$$
z_{n} \leqq z_{n+1}, p_{n} \leqq p_{n+1}, q_{n} \leqq q_{n+1} .
$$

The second result is the fact that if $\psi$ is replaced by another function $\bar{\psi}$ with similar properties and, almost everywhere,

$$
\psi \leqq \bar{\psi}, \psi_{x} \leqq \bar{\psi}_{x}, \psi_{y} \leqq \bar{\psi}_{y},
$$

then the corresponding solution $\bar{z}$ satisfies

$$
z \leqq \bar{z}, p \leqq \bar{p}, q \leqq \bar{q} .
$$


Proof. Define sequences of successive approximations as follows: Let

$$
z_{0}(x, y)=\alpha(x, y), u_{0}(x, y)=\beta(x, y), v_{0}(x, y)=\gamma(x, y)
$$

and, for $n \geqq 1$,

$$
\begin{aligned}
& z_{n}(x, y)=\int_{0}^{x} \int_{0}^{y} \varphi\left(s, t, z_{n-1}(s, t), u_{n-1}(s, t), v_{n-1}(s, t)\right) d s d t, \\
& u_{n}(x, y)=\int_{0}^{y} \varphi\left(x, t, z_{n-1}(x, t), u_{n-1}(x, t), v_{n-1}(x, t)\right) d t, \\
& v_{n}(x, y)=\int_{0}^{x} \varphi\left(s, y, z_{n-1}(s, y), u_{n-1}(s, y), v_{n-1}(\mathrm{~s}, y)\right) d s .
\end{aligned}
$$

The functions $z_{n}, u_{n}, v_{n}$ are defined on sets $R, R_{1}, R_{2}$, respectively, which can be taken independent of $n$. The inequalities (7), (8), (9) give the case $n=0$ of

$$
z_{n} \leqq z_{n+1}, u_{n} \leqq u_{n+1}, \quad v_{n} \leqq v_{n+1} .
$$

The cases $n>0$ of these inequalities follow by induction by virtue of the monotony of $\varphi$.

The boundedness of $\varphi$ implies the uniform boundedness of the functions $z_{n}, u_{n}, v_{n}$. Hence, as $n \rightarrow \infty$

$$
z=\lim z_{n}, u=\lim u_{n}, v=\lim v_{n},
$$

exist on $R, R_{1}, R_{2}$, respectively. It is clear from (15) and (19), (20) that

$$
0 \leqq \alpha \leqq z, 0 \leqq \beta \leqq u, 0 \leqq \gamma \leqq v .
$$

Lebesgue's theorem on term-by-term integration under bounded convergence implies

$$
\begin{aligned}
& z(x, y)=\int_{0}^{x} \int_{0}^{y} \varphi(s, t, z(s, t), u(s, t), v(s, t)) d s d t, \\
& u(x, y)=\int_{0}^{y} \varphi(x, t, z(x, t), u(x, t), v(x, t)) d t, \\
& v(x, z)=\int_{0}^{x} \varphi(s, y z(s, y), u(s, y), v(s, y)) d s .
\end{aligned}
$$

It is clear that $z_{y}=u, z_{y}=v$ almost everywhere. Thus the assumptior on $\varphi$ concerning (3) shows that $z \equiv u \equiv v \equiv 0$. Lemma 1 follows from (21).

4. Proof of $(*)$. (i). Let $z(x, y)$ be a solution of (1). There exis 1 functions $u(x, y), v(x, y)$ defined on sets $R_{1}, R_{2}$, respectively, such that

$$
\begin{aligned}
& z(x, y)=\sigma(x)+\tau(y)-z_{0}+\int_{0}^{x} \int_{0}^{y} f(s, t, z(s, t), u(s, t), v(s, t) d s d t, \\
& u(x, y)=\sigma_{x}(x)+\int_{0}^{y} f(x, t, z(x, t), u(x, t), v(x, t)) d t,
\end{aligned}
$$




$$
v(x, y)=\tau_{y}(y)+\int_{0}^{x} f\left(s, y, z(s, y), z_{x}(s, y), z_{y}(s, y)\right) d s,
$$

and the relations $u=z_{x}$ and $v=z_{y}$ hold almost everywhere. In order to see this, note that almost everywhere on $R$,

$$
\begin{aligned}
& z_{x}(x, y)=\sigma_{x}(x)+\int_{0}^{y} f\left(x, t, z(x, t), z_{x}(x, t), z_{y}(x, t)\right) d t \\
& z_{y}(x, y)=\sigma_{y}(y)+\int_{0}^{x} f\left(s, y, z(s, y), z_{x}(s, y), z_{y}(s, y)\right) d s,
\end{aligned}
$$

The expressions on the right side of these equations are defined for $(x, y)$ on sets $R_{1}, R_{2}$, respectively. Define $u(x, y), v(x, y)$ to be these expressions on $R_{1}, R_{2}$. In particular $z_{x}=u$ and $z_{y}=v$ almost everywhere. Hence (26), (27) hold on (possibly different) sets $R_{1}, R_{2}$. Clearly (25) is valid for all $(x, y)$ on $R$.

(ii). Uniqueness in $(*)$. Suppose that (1) possesses two solutions $z=z_{1}(x, y), z_{2}(x, y)$ on $R$. Let $u_{1}(x, y), v_{1}(x, y)$ and $u_{2}(x, y), v_{2}(x, y)$ be the functions associated with $z_{1}, z_{2}$ by (i). Let $\alpha=\left|z_{1}-z_{2}\right|, \beta=\left|u_{1}-u_{2}\right|$, $\gamma=\left|v_{1}-v_{2}\right|$. If the relations (25) for $z=z_{1}, z_{2}$ are subtracted, it is seen that the inequality (2) for $f$ implies (7). Similarly (26), (27) imply (8), (9) respectively.

The functions $\alpha, \beta, \gamma$ satisfy the assumptions of Lemma 1 . Hence the uniqueness assertion in $(*)$ follows from Lemma 1.

(iii). Existence and successive approximations. Let $z_{0}(x, y), z_{1}(x, y)$, ... be the successive approximations defined by (4). Corresponding to each $z_{n}(x, y)$, it is possible to introduce functions $u_{n}(x, y), v_{n}(x, y)$ defined on sets $R_{1}, R_{2}$, respectively, and satisfying $u_{0}=\sigma_{x}(x), v_{0}=\tau_{y}(y)$,

$$
\begin{aligned}
z_{n}(x, y)=\sigma(x) & +\tau(y)-z_{0} \\
& +\int_{0}^{x} \int_{0}^{y} f\left(s, t, z_{n-1}(s, t), u_{n-1}(s, t), v_{n-1}(s, t)\right) d s d t,
\end{aligned}
$$

$$
u_{n}(x, y)=\sigma_{x}(x)+\int_{0}^{y} f\left(x, t, z_{n-1}(x, t), u_{n-1}(x, t), v_{n-1}(x, t)\right) d t,
$$

$$
v_{n}(x, y)=\tau_{y}(y)+\int_{0}^{x} f\left(s, y, z_{n-1}(x, t), u_{n-1}(s, y), v_{n-1}(x, t)\right) d s .
$$

The sets $R_{1}, R_{2}$ can be assumed to be independent of $n$.

Let $Z_{m n}=\left|z_{m}-z_{n}\right|, U_{m n}=\left|u_{m}-u_{n}\right|, V_{m n}=\left|v_{m}-v_{n}\right|$ and

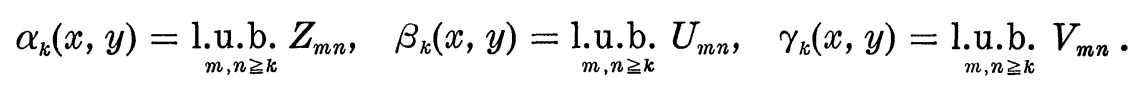

It is clear that $Z_{m n}, U_{m n}, V_{m n}$ are uniformly Lipschitz continuous with respect to $(x, y), x, y$, respectively, and that a corresponding statement holds for $\alpha_{k}, \beta_{k}, \gamma_{k}$.

By subtracting the relation $\left(28_{n}\right)$ from $\left(28_{n-1}\right)$ and using the inequal- 
ity (2) for $f$, it is seen that

$$
Z_{m m}(x, z) \leqq \int_{0}^{x} \int_{0}^{y} \varphi\left(s, t, Z_{m-1 \quad n-1}(s, t), U_{m=1 \quad n-1}(s, t), V_{m-1 \quad n-1}(s, t)\right) d s d t .
$$

Thus, if $m, n \geqq k$, the monotony of $\varphi$ shows that

$$
Z_{m n}(x, y) \leqq \int_{0}^{x} \int_{0}^{y} \varphi\left(s, t, \alpha_{k-1}(s, t), \beta_{k-1}(s, t), \gamma_{k-1}(s, t)\right) d s d t
$$

Hence

$$
\alpha_{k}(x, y) \leqq \int_{0}^{x} \int_{0}^{y} \varphi\left(s, t, \alpha_{k-1}(s, t), \beta_{k-1}(s, t), \gamma_{k-1}(s, t)\right) d s d t
$$

Similarly

$$
\begin{aligned}
& \beta_{k}(x, y) \leqq \int_{0}^{y} \varphi\left(x, t, \alpha_{k-1}(x, t), \beta_{k-1}(x, t), \gamma_{k-1}(x, t)\right) d t, \\
& \gamma_{k}(x, y) \leqq \int_{0}^{x} \varphi\left(s, y, \alpha_{k-1}(s, y), \beta_{k-1}(s, y), \gamma_{k-1}(s, y)\right) d s .
\end{aligned}
$$

By (31), the sequences $\left\{\alpha_{k}(x, y)\right\},\left\{\beta_{k}(x, y)\right\},\left\{\gamma_{k}(x, y)\right\}$ are non-increasing (and non-negative). Let $\alpha(x, y), \beta(x, y), \gamma(x, y)$ denote the respective limits of these sequence, The Lipschitz continuity of $\alpha_{k}, \beta_{k}, \gamma_{k}$ is preserved under the limiting process. Lebesgue's theorem on term-byterm integration under bounded convergence gives the inequalities (7), (8), (9). Hence Lemma 1 shows that $\alpha \equiv 0, \beta \equiv 0, \gamma \equiv 0$ on $R, R_{1}, R_{2}$, respectively. This implies the existence of the functions $z=\lim z_{n}$, $u=\lim u_{n}, v=\lim v_{n}$ on $R_{1}, R_{2}$, as $n \rightarrow \infty$, satisfying (25), (26), (27). It is clear that the limit function $z(x, y)$ is a solution of (1).

Finally, the equicontinuity of the functions $z_{n}(x, y)$ (implied by their uniform Lipschitz continuity) shows that $z(x, z)$ is the uniform limit of the $z_{n}(x, y)$. This proves $(*)$.

5. Lemma for $(* *)$. The proof of $(* *)$ will depend on the following lemma:

Lemma 2. Let $\alpha(x, y), \beta(x, y), \gamma(x, y)$ be non-negative, measurable functions defined on $R, R_{1}, R_{2}$, respectively, so that $\alpha$ is continuous, $\beta$ is uniformly Lipschitz continuous with respect to $y$ and $\gamma$ is uniformly Lipschitz continuous with respect to $x$. Furthermore, assume that

$$
\alpha(x, y) / x y \rightarrow 0 \text { as } 0<x y \rightarrow 0
$$

and that, uniformly with respect to $x$ and $y$, respectively,

$$
\beta(x, y) / y \rightarrow 0 \text { as } y \rightarrow 0 \text { and } \gamma(x, y) / x \rightarrow 0 \text { as } x \rightarrow 0 \text {. }
$$

Finally, suppose that

$$
\begin{array}{r}
\alpha(x, y) \leqq \int_{0}^{x} \int_{0}^{y}\left\{c_{1}(s, t) \alpha(s, t) / s t+c_{2}(s, t) \beta(s, t) / t\right. \\
\left.+c_{3}(s, t) \gamma(s, t) / s\right\} d s d t,
\end{array}
$$




$$
\begin{aligned}
& \beta(x, y) \leqq \int_{0}^{y}\left\{c_{1}(x, t) \alpha(x, t) / x t+c_{2}(x, t) \beta(x, t) / t\right. \\
&\left.+c_{3}(x, t) \gamma(x, t) / x\right\} d t \\
& \gamma(x, y) \leqq \int_{0}^{x}\left\{c_{1}(s, y) \alpha(s, y) / s y+c_{2}(s, y) \beta(s, y) / y\right. \\
& \\
&\left.\quad+c_{3}(s, y) \gamma(s, y) / s\right\} d s,
\end{aligned}
$$

where $c_{1}, c_{2}, c_{3}$ are as in the first part of (**). Then $\alpha \equiv \beta \equiv \gamma \equiv 0$.

Proof. By (32), if $\alpha(x, y) / x y$ is defined as 0 when $x y=0$, it becomes a continuous function on $R$. Hence, it assumes its maximum $M_{1}$ at some point $\left(x^{1}, y^{1}\right) \in R$. Let $M_{2}=1$.u.b. $\beta(x, y) / y$ and $M_{3}=1$.u.b. $\gamma(x, y) / x$ for $(x, y) \in R$.

Note that there exist numbers $M_{j k}$, where $j, k=1,2,3$, satisfying

$$
M_{j k} \geqq 0 \text { and } \sum_{k=1}^{3} M_{j k}=1 \quad \text { for } j=1,2,3 \text {, }
$$

and

$$
M_{\jmath} \leqq \sum_{k=1}^{3} M_{j k} M_{k}
$$

If $M_{1} \neq 0$, then $M_{1}=\alpha\left(x^{1}, y^{1}\right) / x^{1} y^{1}$ holds for some point $\left(x^{1}, y^{1}\right)$ of $R$ with $x^{1} y^{1}>0$. In this case, $\left(38_{1}\right)$ follows from $(34)$ with $(x, y)=\left(x^{1}, y^{1}\right)$ if

$$
M_{1 k}=\left(x^{1} y^{1}\right)^{-1} \int_{0}^{x^{1}} \int_{0}^{y^{1}} c_{k}(s, t) d s d t
$$

If $M_{1}=0$, let $M_{1 k}=c_{k}(0,0)$.

In order to obtain $\left(38_{2}\right)$, let $\left(x_{j}, y_{j}\right)$, where $j=1,2, \cdots$, be points of $R$ such that $\lim \left(x_{j}, y_{j}\right)=\left(x^{2}, y^{2}\right)$ exists, $\lim \beta\left(x_{j}, y_{j}\right) / y_{j}=M_{2}$ and $\lim \beta\left(x_{j}, y\right)=\beta(y)$ exists uniformly for $0 \leqq y \leqq b$. Then (35) leads to $\left(38_{2}\right)$ with

$$
M_{2 k}=\left(y^{2}\right)^{-1} \int_{0}^{y^{2}} c_{k}\left(x^{2}, t\right) d t \text { or } M_{2 k}=c_{k}\left(x^{2}, 0\right)
$$

according as $y^{2}>0$ or $y^{2}=0$. A relation of the type $\left(38_{3}\right)$ is obtained similarly.

Let $M_{J}=\max \left(M_{1}, M_{2}, M_{3}\right)$. Suppose, if possible, that $M_{J}>0$. Assume, for the moment, that $M_{J}>M_{j}$ if $j \neq J$. Then, by (37) and $\left(38_{J}\right), M_{J J}=1$ and $M_{J k}=0$ for $k \neq J$. But the derivation of $\left(38_{J}\right)$ can then be modified to obtain $M_{J}<M_{J}$. For example, if $J=1$, then $c_{1}(s, t) \equiv 1$ and $c_{2}(s, t)=c_{3}(s, t)=0$ in (34) when $(x, y)=\left(x^{1}, y^{1}\right)$, while $\alpha(s, t) / s t$ is nearly zero for small st, so that one obtains $M_{1}<M_{1}$. Or if $J=2$, then $y^{2}>0$ and $c_{1}\left(x^{2}, t\right)=1, c_{2}\left(x^{2}, t\right)=c_{3}\left(x^{2}, t\right)=0$ for $0 \leqq t$ $\leqq y^{2}$, while the relations

$$
\beta(y) \leqq \int_{0}^{y} \beta(t) d t / t, \quad \beta\left(y^{2}\right) / y^{2}=M_{2}
$$

give $M_{2}<M_{2}$ since $\beta(t) / t$ is nearly 0 for small $t$ by the uniformity of 
the first limit relation in (33).

Similar arguments show that if two or three of the numbers $M_{1}$, $M_{2}, M_{3}$ are equal to $M_{J}>0$, one is led to a contradiction. Hence $M_{J}=0$. This proves the lemma.

6. Proof of (**). (i ). Uniqueness in (**). Let $z=z_{1}(x, y), z_{2}(x, y)$ be two solutions of (1) on $R$. Let $u_{1}(x, y), v_{1}(x, y)$ and $u_{2}(x, y), v_{2}(x, y)$ be the functions associated with them as in the proof of (*). Let $\alpha=\left|z_{1}-z_{2}\right|, \beta=\left|u_{1}-u_{2}\right|, \gamma=\left|v_{1}-v_{2}\right| . \quad$ It will be verified that, as $x$ (or $y) \rightarrow 0$, then, except for sets of measure zero,

$$
\alpha(x, y), \beta(x, y), \gamma(x, y) \rightarrow 0 \text {. }
$$

Consider the case $x \rightarrow 0$. The assertions (41) concerning $\alpha$ and $\gamma$ are clear. In order to verify assertion (41) for the function $\beta$, it will first be shown that if $z=z(x, y)$ is any solution of (1) (say, $z=z_{1}$ or $\left.z=z_{2}\right)$ and if $u(x, y) v(x, y)$ are its associated functions, then

$$
\lim u(x, y)=\rho(y) \text {, as } x \rightarrow 0 \text {, exists uniformly in } y \text {. }
$$

To see this, let $x_{j}$, where $j=1,2,3, \cdots$ be a sequence of $x$ values such that $\lim x_{j}=0$ and $\lim u\left(x_{j}, y\right)=\rho(y)$ exists uniformly as $j \rightarrow \infty$. Putting $x=x_{j}$ in (26) and letting $j \rightarrow \infty$, it is seen that

$$
\rho(y)=\sigma_{x}(+0)+\int_{0}^{y} f\left(0, t, \tau(t), \rho(t), \tau_{y}(t)\right) d t .
$$

We note that $\rho(y)$ is continuous. Furthermore, $\rho(y)$ does not depend on the sequence $x_{1}, x_{2}, \ldots$. Suppose that another sequence leads to a different limit $\bar{\rho}(y) \not \equiv \rho(y)$. By substituting $\bar{\rho}$ for $\rho$ in (43), and subtracting, we get

$$
\begin{aligned}
|\bar{\rho}(y)-\rho(y)| \leqq \int_{0}^{y} \mid f(0, t, \tau(t), \bar{\rho}(t), & \left.\tau_{y}(t)\right) \\
& -f\left(0, t, \tau(t), \rho(t), \tau_{y}(t)\right) / d t .
\end{aligned}
$$

Since $f, \rho, \bar{\rho}$ are continuous and $\rho(0)=\bar{\rho}(0)=\sigma_{x}(+0)$, the integrand of (44) can be made small by making $y$ small. Hence

$$
|\bar{\rho}(y)-\rho(y)| / y \rightarrow 0 \text {, as } y \rightarrow 0 \text {. }
$$

By relation (5),

$$
|\bar{\rho}(y)-\rho(y)|\left|y \leqq y^{-1} \int_{0}^{y} c_{2}(0, t)\right| \bar{\rho}(t)-\rho(t) \mid d t / t,
$$

Using (45) as before, this leads to a contradiction. Hence $\bar{\rho} \equiv \rho$. Therefore every sequence, for which the limit in (42) exists, leads to the same limit. Hence (42) holds.

If $\lim u_{1}(x, y)=\rho_{1}(y)$ and $\lim u_{2}(x, y)=\rho_{2}(y)$, as $x \rightarrow 0$, we can repeat the above argument and obtain $\rho_{1} \equiv \rho_{2}$. This completes the verification of (41). 
We now verify assumptions (32) and (33) of Lemma 2. Consider, for example, the assertion

$$
\beta(x, y) / y \rightarrow 0 \text { as } y \rightarrow 0 .
$$

By putting $u=u_{1}, u_{2}$ in (26) and subtracting we get

$$
\begin{aligned}
\beta(x, y) \leqq \int_{0}^{y} \mid f\left(x, t, z_{1}(x, t),\right. & \left.u_{1}(x, t), v_{1}(x, t)\right) \\
& -f\left(x, t, z_{2}(x, t), u_{2}(x, t), v_{2}(x, t)\right) \mid d t .
\end{aligned}
$$

Now the integrand of (47) can be made small, by making $y$ small, and using (41). This proves (46). The other limits in (32) and (33) are verified similarly. The other assumptions of Lemma 2 are quite straightforward. Therefore $\alpha \equiv \beta \equiv \gamma \equiv 0$. This proves "uniqueness".

(ii). Existence and successive approximations in (**). Let $z_{0}(x, y)$, $z_{1}(x, y), \cdots$, be the successive approximations defined by (4). Corresponding to $z_{n}(x, y)$ it is possible to introduce, as in the proof of $(*)$, functions $u_{n}(x, y), v_{n}(x, y)$ defined on sets $R_{1}, R_{2}$ (independent of $n$ ) and satisfying $u_{0}=\sigma_{x}(x), v_{0}=\tau_{y}(y),\left(28_{n}\right),\left(29_{n}\right)$ and $\left(30_{n}\right)$. Let $Z_{m n}, U_{m n}, V_{m n}$ be defined as in the existence proof $(*)$ above. It will be verified that, given $\varepsilon$, there exists a $\delta(\varepsilon)$ and an $N(\varepsilon)$, such that

$$
Z_{m n}(x, y), U_{m n}(x, y), V_{m n}(x, y)<\varepsilon
$$

for $x<\delta(\varepsilon)$ and for all $m, n>N(\varepsilon)$. A similar statement will be seen to hold when $x$ is replaced by $y$. The assertion (48) concerning $Z_{m n}$ and $V_{m n}$ is clear. In order to verify (48) for the function $U_{m n}$ it will first be shown that

$$
\lim u_{n}(x, y)=h_{n}(y) \text {, as } x \rightarrow 0 \text {, exists uniformly in } y \text { and } n \text {. }
$$

It is easily verified, by induction, that $h_{n}(y)$ exists uniformly in $y$ for fixed $n$, where

$$
h_{n}(y)=\sigma_{x}(+0)+\int_{0}^{y} f\left(0, t, \tau(t), h_{n-1}(y), \tau_{y}(t)\right) d t .
$$

To see the uniformity in $n$, define

$\left(51_{n}\right) \quad \bar{z}_{n}(x, y)=z_{n}(x, y)-\sigma(x)-\tau(y)+z_{0} ; \bar{u}_{n}(y, y)=u_{n}(y, y)-\sigma_{x}(y) ;$

$$
\bar{v}_{n}(x, y)=v_{n}(x, y)-\tau_{y}(y) ;
$$

(52) $g(x, y, z, p, q)=f\left(x, y, z+\sigma(x)+\tau(y)-z_{0}, p+\sigma_{x}(x), q+\tau_{y}(y)\right)$.

For $\bar{u}_{n}$ we define $\bar{h}_{n}$ corresponding to $h$. Clearly $g$ satisfies a condition analogous to $(5), \bar{u}_{0}(x, y)=\bar{h}_{0}(y) \equiv 0$, and

$$
\begin{aligned}
\bar{u}_{n}(x, y) & =\int_{0}^{y} g\left(x, t, \bar{z}_{n-1}(x, t), \bar{u}_{n-1}(x, t), \bar{v}_{n-1}(x, t)\right) d t, n \geqq 1 \\
\bar{h}_{n}(y) & =\int_{0}^{y} g\left(0, t, 0, \bar{h}_{n-1}(t), 0\right) d t, n \geqq 1
\end{aligned}
$$


To prove (49) it suffices to verify that

$$
\lim \bar{u}_{n}(x, y)=\bar{h}_{n}(y) \text {, as } x \rightarrow 0 \text {, exists uniformly in } y \text { and } n \text {. }
$$

By subtracting $\left(54_{n}\right)$ from $\left(53_{n}\right)$, it is seen that

$$
\left|\bar{u}_{n}(x, y)-\bar{h}_{n}(y)\right| \leqq \int_{0}^{y}\left\{\left|g_{1}-g_{2}\right|+\left|g_{2}-g_{3}\right|\right\} d t
$$

where $g_{1}=g\left(x, t, \bar{z}_{n-1}(x, t), \bar{u}_{n-1}(x, t), \bar{v}_{n-1}(x, t)\right), g_{2}=g\left(0, t, 0, \bar{u}_{n-1}(x, t), 0\right)$ and $g_{3}=g\left(0, t, 0, \bar{h}_{n-1}(t), 0\right)$. We note that, given $\varepsilon>0$, there exists a $\delta(\varepsilon)$ such that $\left|g_{1}-g_{2}\right|<\varepsilon$ if $x<\delta$ for all $y$ and $n$. Hence, noting (5),

$$
\left|\bar{u}_{n}(x, y)-\bar{h}_{n}(z)\right| \leqq \int_{0}^{y}\left\{\varepsilon+t^{-1} c_{2}(0, t)\left|\bar{u}_{n-1}(x, t)-\bar{h}_{n-1}(t)\right|\right\} d t .
$$

By continuity, because of $(6 *), c_{2}(0, t)<1$ for small $t>0$. Hence there exists a number $\theta, 0<\theta<1$, such that

$$
\int_{0}^{y} c_{2}(0, t) d t \leqq \theta y \text { for } 0<y \leqq b .
$$

A simple induction shows that

$$
\left|\bar{u}_{n}(x, y)-\vec{h}_{n}(y)\right| \leqq\left(1-\theta^{n}\right) \varepsilon y /(1-\theta) \leqq b \varepsilon /(1-\theta) .
$$

This proves (55). Hence (49) is established.

Next we note that $h_{n}(y), n=0,1,2, \cdots$, are the successive approximations for the initial value problem

$$
d w / d t=F(t, w), w(0)=\sigma_{x}(+0),
$$

where $F(t, w)=f\left(0, t, \tau(t), w, \tau_{y}(t)\right)$ is bounded, measurable and continuous in $w$ (for almost all fixed $t$ ). By (5),

$$
|F(t, w)-F(t, \bar{w})| \leqq|w-\bar{w}| / t .
$$

Note that the existence of $\tau_{y}(+0)$ implies that $F(t, w) \rightarrow F(0, w)=$ $f\left(0,0, \tau(0), w, \tau_{y}(+0)\right)$ as $t \rightarrow+0$. The proof of the main theorem in [8] shows that these successive approximations converge uniformly, (60) being Nagumo's uniqueness condition (cf. [5], p. 97). Hence

$$
\lim h_{n}(y)=h(y) \text {, exists uniformly in } y \text { as } n \rightarrow \infty \text {. }
$$

Now (61) and (49) together give (48) for $U_{m n}(x, y)$. Hence (48) is established.

By an argument similar to that used in verifying (46) it is seen that, given $\varepsilon>0$, there exists $\delta(\varepsilon)$ such that

$$
\begin{aligned}
& (x y)^{-1} Z_{m n}(x, y)<\varepsilon \text { for } x y<\delta(\varepsilon) \text { and for } m, n>N(\varepsilon) \\
& x^{-1} U_{m n}(x, y)<\varepsilon \text { for } x<\delta(\varepsilon) \text { and for } m, n>N(\varepsilon) \\
& y^{-1} V_{m n}(x, y)<\varepsilon \text { for } y<\delta(\varepsilon) \text { and for } m, n>N(\varepsilon) .
\end{aligned}
$$

Now defining $\alpha_{k}, \beta_{k}, \gamma_{k}$ as in (31), we note that we can substitute 
them for $Z_{m n}, U_{m n}, V_{m n}$, respectively, in (62) changing $m, n>N(\varepsilon)$ to $k>N(\varepsilon)$. Proceeding as in the analogous section of the proof of theorem (*), we conclude that $\alpha, \beta, \gamma$, satisfy (34), (35) and (36), also (32) and (33). Therefore, by Lemma 2 , the successive approximations converge uniformly to a solution of (1).

7. Counter-examples. (a). Let $a=b=1,1+\varepsilon=\delta^{2}, \varepsilon>0, \delta>1$. Let $f(x, y, z, p, q)$ be independent of $p, q$ and defined by

$$
f(x, y, z, p, q)= \begin{cases}0 & \text { if }(x, y) \in R, z \leqq 0 \\ (1+\varepsilon) z / x y & \text { if }(x, y) \in R, 0<z<(x y)^{\delta}, \\ (1+\varepsilon)(x y)^{\delta-1} & \text { if }(x, y) \in R,(x y)^{\delta} \leqq z .\end{cases}
$$

Then $f(x, y, z, p, q)$ is continuous and satifies (5) for $c_{1}(x, y)=1+\varepsilon$, (and $\left.c_{2}=c_{3} \equiv 0\right)$. Let $\sigma(x)=\tau(y) \equiv 0$. Then (1) has an infinity of solutions, namely, $z=c(x y)^{\delta}$, where $0<c<1$.

(b). Let $a=b=1, R^{0}=\{(x, y): 0<x, y \leqq 1\}, 1+\varepsilon=\delta^{2}, \varepsilon>0$, $\delta>0$ and

$$
f(x, y, z, p, q)= \begin{cases}0 & \text { if } x=0, y=0, \\ (x y)^{\delta-1} & \text { if }(x, y) \in R^{0}, z<0, \\ (x y)^{\delta-1}-(1+\varepsilon) z / x y & \text { if }(x, y) \in R^{0}, 0 \leqq z \leqq(x y)^{0}, \\ -\varepsilon(x y)^{\delta-1} & \text { if }(x, y) \in R^{0},(x y)^{\delta}<z .\end{cases}
$$

Then $f(x, y, z, p, q)$ satisfies the same relation (5) as in example (a). However, in (4), $z_{2 n}=0, z_{2 n+1}=(x y)^{\delta} / \delta^{2}$, so that successive approximations (4) do not converge.

\section{REFERENCES}

1. E. A. Coddington and N. Levinson, Uniqueness and the convergence of successive approximations, J. Indian Math. Soc,. 16 (1952), 75-81.

2. F. Guglielmino, Sulla risoluzione del problema de Darboux per I'equazione $s=f(x, y, z$,$) ,$ Bollettino della Unione Matematica Italiana, 13 (1958), 308-318.

3. P, Hartman and A. Wintner, On hyperbolic partial differential equations, Amer. J. Math. 74 (1952), 832-864.

4. E. K. Haviland, $A$ note on the convergence of the successive approximations to the solution of an ordinary differential equation, Amer. J. Math. 54 (1932), 632-634.

5. E. Kamke, Differentialgleichungen reeler Funktionen, Leipzig (1930).

6. J. Kisynski, Sur I'existence et l'unicité des solutions des problemes classiques relatifs á l'equation $s=F(x, y, z, p, q$,), Annales Mariae Curie-Sklodowska, 11 (1957), 73-112.

7. O. Perron, Eine hinreichende Bedingung für die Unität der Lösung von Differentialgleichungen erster Ordnnug, Mathematische Zeitschrift, 28 (1928), 216-219.

8. B. Viswanatham, The general uniqueness theorem and successive approximations, J.Indian Math. Soc., 16 (1952), 69-74.

THE JOHNS HOPKINS UNIVERSITY 


\section{PACIFIC JOURNAL OF MATHEMATICS}

\section{EDITORS}

David Gilbarg

Stanford University

Stanford, California

F. H. Brownell

University of Washington

Seattle 5, Washington
A. L. Whiteman

University of Southern Californıa Los Angeles 7, California

L. J. PaIge

University of California

Los Angeles 24, California

\section{ASSOCIATE EDITORS}
E. F. BECKENBACH
T. M. CHERRY
D. DERRY

E. HEWITT
A. HORN
L. NACHBIN
M. OHTSUKA

H. L. ROYDEN

M. M. SCHIFFER
E. SPANIER

E. G. STRAUS

F. WOLF

\section{SUPPORTING INSTITUTIONS}

\author{
UNIVERSITY OF BRITISH COLUMBIA \\ CALIFORNIA INSTITUTE OF TECHNOLOGY \\ UNIVERSITY OF CALIFORNIA \\ MONTANA STATE UNIVERSITY \\ UNIVERSITY OF NEVADA \\ NEW MEXICO STATE UNIVERSITY \\ OREGON STATE COLLEGE \\ UNIVERSITY OF OREGON \\ OSAKA UNIVERSITY \\ UNIVERSITY OF SOUTHERN CALIFORNIA
}

\author{
STANFORD UNIVERSITY \\ UNIVERSITY OF TOKYO \\ UNIVERSITY OF UTAH \\ WASHINGTON STATE COLLEGE \\ UNIVERSITY OF WASHINGTON \\ AMERICAN MATHEMATICAL SOCIETY \\ CALIFORNIA RESEARCH CORPORATION \\ HUGHES AIRCRAFT COMPANY \\ SPACE TECHNOLOGY LABORATORIES \\ NAVAL ORDNANCE TEST STATION
}

Mathematical papers intended for publication in the Pacific Journal of Mathematics should be typewritten (double spaced), and the author should keep a complete copy. Manuscripts may be sent to any one of the four editors. All other communications to the editors should be addressed to the managing editor, L. J. Paige at the University of California, Los Angeles 24, California.

50 reprints per author of each article are furnished free of charge; additional copies may be obtained at cost in multiples of 50 .

The Pacific Journal of Mathematics is published quarterly, in March, June, September, and December. The price per volume (4 numbers) is $\$ 12.00$; single issues, $\$ 3.50$. Back numbers are available. Special price to individual faculty members of supporting institutions and to individual members of the American Mathematical Society: $\$ 4.00$ per volume; single issues, $\$ 1.25$.

Subscriptions, orders for back numbers, and changes of address should be sent to Pacific Journal of Mathematics, 2120 Oxford Street, Berkeley 4, California.

Printed at Kokusai Bunken Insatsusha (International Academic Printing Co., Ltd.), No. 6, 2-chome, Fujimi-cho, Chiyoda-ku, Tokyo, Japan.

PUBLISHED BY PACIFIC JOURNAL OF MATHEMATICS, A NON-PROFIT CORPORATION

The Supporting Institutions listed above contribute to the cost of publication of this Journal, but they are not owners or publishers and have no responsibility for its content or policies. 


\section{Pacific Journal of Mathematics}

\section{Vol. 10, No. $2 \quad$ October, 1960}

Maynard G. Arsove, The Paley-Wiener theorem in metric linear spaces ........

Robert (Yisrael) John Aumann, Acceptable points in games of perfect

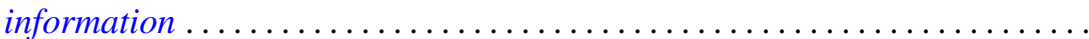

A. V. Balakrishnan, Fractional powers of closed operators and the semigroups generated by them ... . . . . . . . . . . . . . . . . . . . . . . . . . . . . 419

Dallas O. Banks, Bounds for the eigenvalues of some vibrating systems . . . . . 439

Billy Joe Boyer, On the summability of derived Fourier series . . . . . . . . . . . 475

Robert Breusch, An elementary proof of the prime number theorem with

remainder term ...................................

Edward David Callender, Jr., Hölder continuity of $n$-dimensional

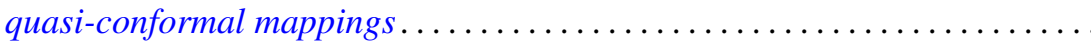

L. Carlitz, Note on Alder's polynomials ......................... 517

P. H. Doyle, III, Unions of cell pairs in $E^{3} \ldots \ldots \ldots \ldots \ldots \ldots \ldots \ldots \ldots \ldots \ldots \ldots . \ldots 21$

James Eells, Jr., A class of smooth bundles over a manifold . . . . . . . . . . . . 525

Shaul Foguel, Computations of the multiplicity function . . . . . . . . . . . . . . 539

James G. Glimm and Richard Vincent Kadison, Unitary operators in

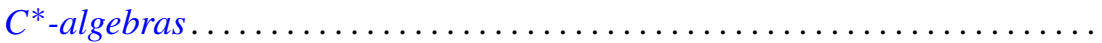

Hugh Gordon, Measure defined by abstract $L_{p}$ spaces . . . . . . . . . . . 557

Robert Clarke James, Separable conjugate spaces ....................

William Elliott Jenner, On non-associative algebras associated with bilinear forms

Harold H. Johnson, Terminating prolongation procedures

John W. Milnor and Edwin Spanier, Two remarks on fiber homotopy type .

Donald Alan Norton, A note on associativity . .

Ronald John Nunke, On the extensions of a torsion module.

Joseph J. Rotman, Mixed modules over valuations rings . . . . .

A. Sade, Théorie des systèmes demosiens de groupoï des . .

Wolfgang M. Schmidt, On normal numbers . .

661

Berthold Schweizer, Abe Sklar and Edward Oakley Thorp, The metrization of

statistical metric spaces

John P. Shanahan, On uniqueness questions for hyperbolic differential

equations

A. H. Stone, Sequences of coverings

Edward Oakley Thorp, Projections onto the subspace of compact operators

L. Bruce Treybig, Concerning certain locally peripherally separable spaces

Milo Wesley Weaver, On the commutativity of a correspondence and a

permutation

David Van Vranken Wend, On the zeros of solutions of some linear complex

differential equations. 